\title{
Lab-facility of HEP detectors and related technology for training, development, fabrication, applications and innovation
}

\author{
J.F. Castaño*, L.F. Castañeda, R.M. Gutierrez, A.I. Hernandez, H. Yepes-Ramirez \\ CICBA - Centro de Investigaciones en Ciencias Básicas y Aplicadas \\ Universidad Antonio Nariño. Cra. 3 Este No. 47 A - 15, Bloque 4 - Piso 3 \\ Bogotá D.C., Colombia \\ E-mail: jfcastanof@uan.edu.co
}

\begin{abstract}
We present a Lab-facility of HEP detectors built at Universidad Antonio Nariño (UAN), initially supported in 2012 by a RD51-CERN common funding project, latter improved with UAN selffunding, technical resources and knowledge provided in the frame of the NEXT experiment where UAN is the only Latin-American collaborator. We show the structure and resources of the Lab, as well as a summary of the main results concerning education and innovation.
\end{abstract}

38th International Conference on High Energy Physics

3-10 August 2016

Chicago, USA

\footnotetext{
* Speaker.
} 


\section{Introduction}

Detectors Laboratory (Laboratorio de Detectores) of the UAN is a Lab-facility for training, development, fabrication, applications and innovation of HEP detectors, particularly, Silicon Photomultiplier (SiPM) and Micro-Pattern Gas Detector (MPGD), as well as their related technologies. The goal of the UAN Detectors Laboratory is the training of young researchers and $\mathrm{PhD}$ students in particle physics detectors, and the support of a PhD program in Applied Science at UAN (unique in Colombia). In addition, the Lab looks to increase the critical mass in such important scientific and technological advances, in particular for Latin America, with new capacities for detectors development, fabrication, quality control and detectors characterization for particle physics and new applications in other disciplines and social sectors.

RD51 is a scientific collaboration with participants from five continents. Its main goal is the basicor application-oriented $\mathrm{R} \& \mathrm{D}$ with different gaseous detectors concepts, focused to the advanced technological development and application of MPGDs (e.g., Gas Electron Multiplier or "GEM") [1]. UAN participates actively in the RD51 collaboration since 2010. NEXT is a particle physics experiment aiming to detect the neutrinoless double beta decay using a High-Pressure Gas Xenon Time Projection Chamber with separated-optimized functions for calorimetry and tracking [2]. UAN participates in the NEXT collaboration since 2012 through data analysis, electronics design and SiPM characterization.

\section{UAN Detectors Laboratory}

The UAN Detectors Laboratory (Figure 1) is divided into three different areas: Research I, Research II and Training.

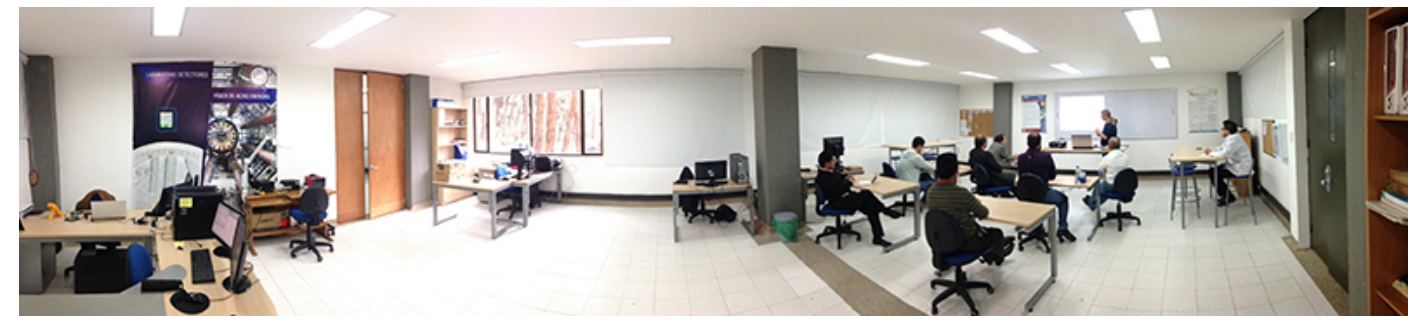

Figure 1: A $180^{\circ}$ view of Research I (left) and Training (right) Areas at the UAN Detectors Laboratory.

Figure 1-left shows the $45 \mathrm{~m}^{2}$ Research Area I, dedicated to basic experimentation for young researchers and $\mathrm{PhD}$ students. This area can support up to 6 researchers workstations and includes in turn the workspace for the technical and administrative staff. Research Area I includes a small section for Printed Circuit Board design and fabrication, with UV equipment, reflow oven, motortool and welding controlled systems. Figure 1-right shows the $35 \mathrm{~m}^{2}$ area dedicated for training/instruction in radiation and particle detectors for young researchers and $\mathrm{PhD}$ students. The training area has technical resources such as HD projector, computers, smart and conventional blackboard, as well as personal desktops and tables.

Figure 2 shows a section of the $28 \mathrm{~m}^{2}$ Research Area II (behind Research Area I back door), with specialized equipment for detectors characterization. Some of the available equipment includes 


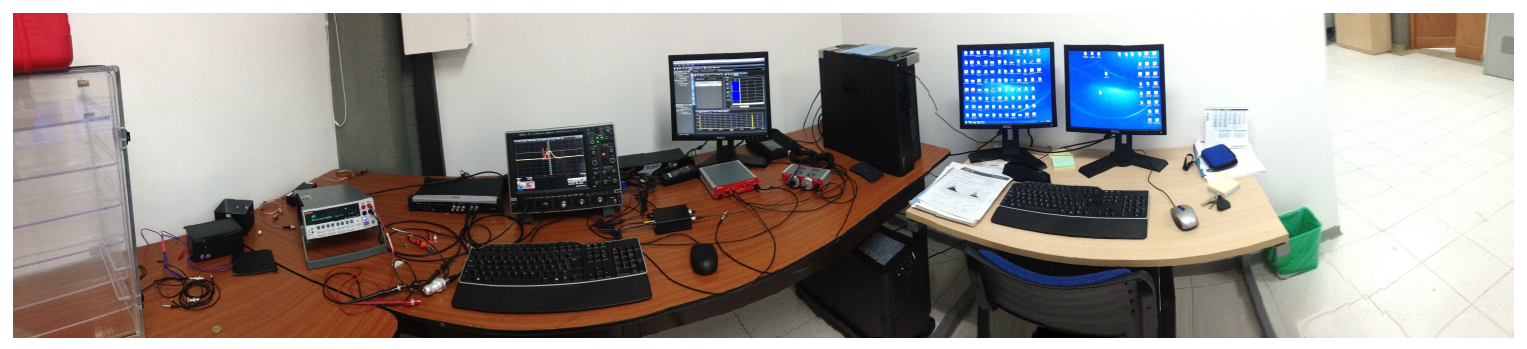

Figure 2: Section of Research Area II and some available equipment for detectors operation.

high voltages sources, SiPM and MPGDs characterization setups, laminar flux cabinet, workstations, among others resources. Additionally, the Lab owns other technical tools and portable measurements devices such as multimeters and luxmeters. There is, in addition, a $4 \mathrm{~m}^{2}$ clean room into Research Area II fullfilling ISO 5 standards, and preparation and dressing section following ISO 7 regulations. The clean workspace (ISO 5) has 30 seconds recovery time and high performance HEPA filters in order to guarantee the best cleaning conditions, required for detectors assembly and other tasks.

The UAN Detectors Laboratory is a main branch of the Centro de Investigaciones en Ciencias Básicas y Aplicadas (CICBA) at UAN. It hosts the best ranked research groups of the UAN and some top groups in Colombia, as the Complex Systems (A1 rank) and High Energy Physics (B rank) groups.

\section{Education and Innovation}

One of the goals of the UAN Detectors Laboratory is to provide an environment for young researchers and PhD students looking to develop their R\&D projects, as well as to improve their skills through a guided education program by high-level staff from physicists to engineers. The Lab, up to now, has supported different education and internship activities as:

- $2 \mathrm{PhD}$ thesis in the Applied Science program at UAN, focused to SiPM application in Visible Light Communication (VLC) [3] [4] [5] [6] [7], almost concluded. This work is actually under patent and other intellectual property protection process.

- $1 \mathrm{PhD}$ thesis in the Applied Science program at UAN, focused to the GEM-foils quality testing by image processing techniques, almost concluded.

- $1 \mathrm{PhD}$ thesis in the Applied Science program at UAN, focused to GEM applications in industry, an ongoing work.

- 1 undergraduate thesis in Electronics Engineering program at UAN, focused to the design and implementation of an experimental setup for SiPM characterization with temperature and light source control, concluded in 2015. 
- 1 German undergraduate student internship during 3 months supported by the German DAAD program, where a software tool for detectors data analysis was developed, currently an enhancement of the Lab experimental analysis frameworks.

- 2 one-year internship of young researchers funded by Colciencias (Colombian Reasearch Council) and UAN.

- 2 internships for UAN undergraduate students for introduction to research (6 months).

\section{Outlook}

The UAN Detectors Laboratory will start a 50k USD grant by Colciencias, enforceable between 2016 and 2018. This supports the continuity to the development of VLC technology with SiPM as optical receiver, totally an innovation and original product of the UAN.

The Lab has presented a proposal for a new 2 Young Researchers incorporation since 2017 until 2018 and 2 new PhD students since 2017 until 2020, funded by Colciencias, a constant-growing policy involving UAN. The Lab continues improving its performance regarding infrastructure, instrumentation and human resources, and collaboration within the RD51 and NEXT programs.

\section{References}

[1] The RD51 collaboration. http://rd51-public.web.cern.ch/RD51-Public/

[2] The NEXT collaboration. http://next.ific.uv.es/next/

[3] R.M. Gutierrez et.al. The silicon photomultiplier as a metasystem with electronics designed as metadevice for a new receiver-emitter in visible light communications. Proc. SPIE 9544, Metamaterials, Metadevices, and Metasystems 2015, 95441P (September 1, 2015); doi:10.1117/12.2190161.

[4] Castañeda, L.F. et.al. A cellular architecture with new SiPM-based devices for an efficient VLC network. Prepint.

[5] Castañeda, L.F. et.al. A new VLC-receiver based on SiPM. Prepint.

[6] Castañeda, L.F. et.al. Advanced Study of SiPM Detectors for VLC Networks. Prepint.

[7] Castañeda, L.F. et.al. New communication protocol and devices with SiPM-based receiver useful for an efficient VLC network. Prepint. 